\title{
Constraints on the Primordial Black Hole Abundance from the First Advanced LIGO Observation Run Using the Stochastic Gravitational-Wave Background
}

\author{
Sai Wang, ${ }^{1, *}$ Yi-Fan Wang, ${ }^{1, \dagger}$ Qing-Guo Huang, ${ }^{2,3,4, \ddagger}$ and Tjonnie G. F. $\mathrm{Li}^{1}{ }^{1} \S$ \\ ${ }^{1}$ Department of Physics, The Chinese University of Hong Kong, Shatin, N.T., Hong Kong \\ ${ }^{2}$ CAS Key Laboratory of Theoretical Physics, Institute of Theoretical Physics, \\ Chinese Academy of Sciences, Beijing 100190, China \\ ${ }^{3}$ School of Physical Sciences, University of Chinese Academy of Sciences, No. 19A Yuquan Road, Beijing 100049, China \\ ${ }^{4}$ Synergetic Innovation Center for Quantum Effects and Applications, \\ Hunan Normal University, Changsha 410081, China
}

\begin{abstract}
Advanced LIGO's discovery of gravitational-wave events is stimulating extensive studies on the origin of binary black holes. Assuming that the gravitational-wave events can be explained by binary primordial black hole mergers, we utilize the upper limits on the stochastic gravitational-wave background given by Advanced LIGO as a new observational window to independently constrain the abundance of primordial black holes in dark matter. We show that Advanced LIGO's first observation run gives the best constraint on the primordial black hole abundance in the mass range $1 M_{\odot} \lesssim M_{\mathrm{PBH}} \lesssim 100 M_{\odot}$, pushing the previous microlensing and dwarf galaxy dynamics constraints tighter by 1 order of magnitude. Moreover, we discuss the possibility to detect the stochastic gravitational-wave background from primordial black holes, in particular from subsolar mass primordial black holes, by Advanced LIGO in the near future.
\end{abstract}

Introduction.-During the first Advanced LIGO observing run, two gravitational wave (GW) events, GW150914 and GW151226, were observed [1,2]. Both GW signals are found to be consistent with the mergers of black holes (BHs). GW150914 originated from two relatively heavy coalescing BHs with masses of $36_{-4}^{+5} M_{\odot}$ and $29_{-4}^{+4} M_{\odot}[1]$, while GW151226 originated from two coalescing BHs with masses of $14_{-4}^{+8} M_{\odot}$ and $7_{-2}^{+2} M_{\odot}[2]$. The local merger rate of the binary black hole $(\mathrm{BBH})$ mergers has been inferred to be $3.4_{-2.8}^{+8.8} \mathrm{Gpc}^{-3} \mathrm{yr}^{-1}$ for GW150914, and $36_{-30}^{+95} \mathrm{Gpc}^{-3} \mathrm{yr}^{-1}$ for GW151226 [3], where the uncertainties are given at a $90 \%$ confidence level. These discoveries robustly demonstrate that BBHs indeed exist and can merge within the age of the Universe.

The origin of these BHs and the formation mechanism of a $\mathrm{BBH}$ are still under debate. Besides an astrophysical origin [4-7], the possibility that these $\mathrm{BHs}$ are of a primordial origin and constitute a fraction of dark matter is also considered [8-14]. The primordial black hole $(\mathrm{PBH})$ abundance in dark matter has been constrained from a variety of observations, including microlensing events caused by massive astrophysical compact halo objects [15-18], the gas accretion effect of PBHs on cosmic microwave background (CMB) [19] and the nondetection of a third-order Shapiro time delay using a pulsar timing array [20] (see Ref. [21] and references therein). Nevertheless, a primordial origin of GW150914 and GW151226 has not been ruled out.

\footnotetext{
*wangsai@itp.ac.cn

†yfwang@phy.cuhk.edu.hk

¥ huangqg@itp.ac.cn

$\S$ tgfli@phy.cuhk.edu.hk
}

Currently, the nature of dark matter is still uncertain. There is no definitive evidence for weakly interacting massive particles (WIMPs), a prime candidate for dark matter, from experiments such as the Particle and Astrophysical Xenon Detector (PandaX-II) [22], the Large Underground Xenon dark matter experiment (LUX) [23], the Large Hadron Collider (LHC) [24], the Alpha Magnetic Spectrometer (AMS-02) [25] and the Fermi Large Area Telescope (Fermi LAT) [26]. The situation motivates one to consider dark matter candidates other than WIMPs such as superWIMPs, light gravitinos, hidden dark matter, sterile neutrinos and axions [27]. Amongst these alternatives, $\mathrm{PBHs}$ are also possible candidates of dark matter [21].

PBHs could be produced by direct gravitational collapse of a primordial overdensity in the early Universe, deep in the radiation dominated era [28-32]. At the formation redshift $z_{f}$, the $\mathrm{PBH}$ mass is roughly equal to the horizon mass, namely $M_{\mathrm{BH}} \simeq \frac{4 \pi}{3} \rho_{f}\left(H_{f}^{-1}\right)^{3} \sim$ $30 M_{\odot}\left[4 \times 10^{11} /\left(1+z_{f}\right)\right]^{2}[10]$. Different mechanisms have been proposed to form binary systems from these PBHs. Two PBHs might pass by each other accidentally and then form a binary due to energy loss by gravitational radiation $[8,9]$. To account for the estimated GW event rate, $\mathrm{PBHs}$ need to contribute to most of the dark matter in this model. On the other hand, two nearby PBHs can form a binary due to the tidal force from the third neighboring $\mathrm{PBH}[10,33]$. The $\mathrm{PBH}$ fraction of dark matter in this model can be smaller than that of Refs. [8,9] and still be compatible with the estimated local merger rate from the gravitational-wave detections. The expected local merger rate of binary $\mathrm{PBH}$ mergers for both these models is consistent with Advanced LIGO's estimate [810]. Therefore, the binary $\mathrm{PBH}$ scenario is capable of explaining GW150914 and GW151226. 
The stochastic gravitational-wave background (SGWB) from BBHs is produced from the incoherent superposition of all the merging binaries in the Universe [34-42]. This background is potentially measurable at Advanced LIGO's projected final sensitivity [41]. Recently, the SGWB following the PBH binary formation mechanism in Refs. $[8,9]$ was shown to be difficult to detect by Advanced LIGO detectors given a single-mass spectrum [43]. The amplitude of the SGWB from PBHs could be enhanced if PBHs cluster in subhalos and have a broad mass distribution with the width of the mass distribution $\Delta M \gtrsim 10^{2} M_{\odot}$ [44].

In this work, we utilize the upper limit on the SGWB given by Advanced LIGO as a new observational window to independently constrain the abundance of PBHs in dark matter, and compare it to a variety of other constraining methods mentioned above. Moreover, we consider the SGWB spectra from different PBH masses, particularly from subsolar mass $\mathrm{PBHs}$, and show that the current most stringent constraints on PBH abundance can give a measurable SGWB in upcoming observing runs of Advanced LIGO. The SGWB from PBHs provides a complementary channel to investigate the existence of subsolar mass BHs, which is a smoking gun for PBHs, even if their GW signals are too weak to be resolved individually.

Merger rate of primordial black hole binaries.- We give a brief overview of the formation mechanism of the binary PBH mergers proposed in Ref. [33] and revisited by Refs. $[10,45]$ to study the merger rate of $\mathrm{PBH}$ binaries and the SGWB from PBH binary merger. PBHs are formed deep in the radiation-dominated epoch and decouple from the background when the average energy density of PBHs exceeds the background cosmic energy density. The tidal force from a third $\mathrm{PBH}$ causes a $\mathrm{PBH}$ pair to move along elliptical orbits and finally to merge due to the energy loss via gravitational radiation. Assuming the abundance of PBHs in dark matter to be $f$ (i.e., $\Omega_{\mathrm{PBH}}=f \Omega_{\mathrm{DM}}$ ), and a fixed $\mathrm{PBH}$ mass $M_{\mathrm{PBH}}$, the probability that the coalescence occurs in the cosmic time interval $(t, t+d t)$ is given by

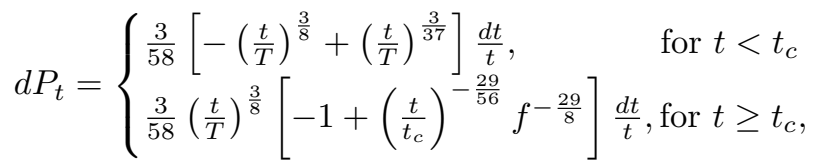

where $T=\frac{3}{170} \frac{c^{5} \bar{x}^{4}}{\left(G M_{\mathrm{PBH}}\right)^{3} f^{4}}$ and $t_{c}=\frac{3}{170} \frac{c^{5} \bar{x}^{4} f^{25 / 3}}{\left(G M_{\mathrm{PBH}}\right)^{3}}$ are constants, $c$ is the speed of light, $G$ is the gravitational constant, and $\bar{x}$ is the physical mean separation of PBHs at the epoch of matter-radiation equality when redshift $z=z_{\text {eq }}[10]$.

The merger rate of $\mathrm{PBH}$ binaries is then given by

$$
R_{\mathrm{PBH}}\left(z ; M_{\mathrm{PBH}}, f\right)=\frac{3 H_{0}^{2}}{8 \pi G} \frac{f \Omega_{\mathrm{DM}}}{M_{\mathrm{PBH}}} \frac{d P_{t}}{d t} .
$$

Here the redshift $z$ is related to the cosmic time $t$ by $t=t_{0}-\frac{1}{H_{0}} \int_{0}^{z} \frac{d z^{\prime}}{\left(1+z^{\prime}\right) E\left(z^{\prime}\right)}$, where $t_{0}$ denotes the age of the Universe and $E(z) \equiv H(z) / H_{0}=$ $\left[\Omega_{\mathrm{r}}(1+z)^{4}+\Omega_{\mathrm{M}}(1+z)^{3}+\Omega_{\Lambda}\right]^{1 / 2}$. Throughout this work, we use the cosmological parameters derived from the 2015 Planck data set [46], i.e., the Hubble constant $H_{0}=67.8 \mathrm{~km} \mathrm{~s}^{-1} \mathrm{Mpc}^{-1}$, the fraction of radiation $\Omega_{r}=$ $9.061 \times 10^{-5}$, the fraction of dark matter $\Omega_{\mathrm{DM}}=0.270$, the fraction of nonrelativistic matter $\Omega_{M}=0.307$ and the fraction of dark energy $\Omega_{\Lambda}=1-\Omega_{M}-\Omega_{\mathrm{r}}$. For the PBH mass spectrum, a narrow spread distribution [4750] and an extended distribution [51, 52] are both considered by early Universe models. However, it has been shown that the inflationary scenario does not favor those with a significantly extended PBH mass distribution [21]. We also find that for a Gaussian PBH mass distribution with a narrow width $\Delta M \sim 1 M_{\odot}$ the resulting SGWB amplitude is negligibly different (less than $1 \%$ between $10-100 \mathrm{~Hz}$ ) from that by assuming a fixed mass distribution. A later work by Ref. [53] that generalized our constraining results also confirmed that assuming a lognormal mass distribution with variance $\sigma \sim O(1)$ would not change the order of magnitude of the upper limits on PBH abundance. Therefore, given the large uncertainties in the PBH mass distribution [21] and aiming to investigate to which extent SGWB can constrain the PBH abundance, we follow Sasaki et al. [10] and use the simplifying assumption that all PBHs have the same mass.

In contrast to binary PBHs, the astrophysical BBH merger rate $R_{\text {astro }}(z)$, which is described in e.g., Ref. [41], peaks at $z=1 \sim 2$, because of the peak in the astrophysical star formation rate [54]. While for the PBH binaries whose mass and local merger rate are consistent with those of GW150914 and GW151226, the merger rate $R_{\mathrm{PBH}}(z)$ keeps rising out to a large redshift (at least $z \sim 30$, see Ref. [55]), due to the fact that PBHs form in the early Universe, and thus have a larger merger rate at high redshift than astrophysical BBHs.

The merger rate as a function of redshift, especially at high redshift, can give us important information about the origin of $\mathrm{BBHs}$, since $R_{\mathrm{PBH}}(z)$ and $R_{\text {astro }}(z)$ behave differently. Recently it has been proposed that Pre-DECIGO (pre-DECihertz laser Interferometer Gravitational-wave Observatory) can determine the origin of GW150914-like BBHs by measuring the mass spectrum and the $z$ dependence of the merger rate [55]. Therefore, future space-based GW detectors, such as LISA [13, 56], DECIGO [57], and BBO [58], may also be used to study the origin of BBHs. The correlation of GW events with galaxy catalogs may also distinguish the origin of BBHs [59].

Stochastic gravitational-wave background energy density spectrum.-Given the merger rate of BBHs, one can 
obtain the SGWB energy density spectrum from

$$
\Omega_{\mathrm{GW}}=\frac{\nu}{\rho_{c}} \frac{d \rho_{\mathrm{GW}}}{d \nu}
$$

where $d \rho_{\mathrm{GW}}$ is the gravitational radiation energy density in the frequency interval $(\nu, \nu+d \nu)$, and $\rho_{c}=3 H_{0}^{2} c^{2} / 8 \pi G$ is the critical energy density of the Universe [60]. For the SGWB produced by binary PBH mergers, $\Omega_{\mathrm{GW}}$ can be expressed as an integral over the redshift, namely

$$
\begin{aligned}
\Omega_{\mathrm{GW}}\left(\nu ; M_{\mathrm{PBH}}, f\right)= & \frac{\nu}{\rho_{c} H_{0}} \int_{0}^{z_{\text {sup }}} \frac{R_{\mathrm{PBH}}\left(z ; M_{\mathrm{PBH}}, f\right)}{(1+z) E(z)} \\
& \times \frac{d E_{\mathrm{GW}}}{d \nu_{s}}\left(\nu_{s}\right) d z,
\end{aligned}
$$

where $d E_{\mathrm{GW}} / d \nu_{s}\left(\nu_{s}\right)$ is the GW energy spectrum of $\mathrm{BBHs}$ coalescence, $\nu_{s}$ is the frequency in the source frame and is related to the observing frequency $\nu$ through $\nu_{s}=(1+z) \nu$. The factor $(1+z)$ on the denominator converts the merger rate from source frame to the observer frame. For this work, we assume an inspiral-mergerringdown energy spectrum with nonprecessing spin correction $[61,62]$. The upper limit of the integration is given by $z_{\text {sup }}=\min \left(z_{\max }, \nu_{\text {cut }} / \nu-1\right)$, where $\nu_{\text {cut }}$ is the cutoff frequency given the energy spectrum of the $\mathrm{BBH}$ and $z_{\max }$ is the maximum redshift predicted by the $\mathrm{PBH}$ model. Since $\mathrm{PBHs}$ are formed in the early Universe, $z_{\max }$ is larger than $\nu_{\text {cut }} / \nu-1$ so that $z_{\text {sup }}$ never takes the value of $z_{\max }$ in the Advanced LIGO sensitive frequency band.

Ref. [43] investigates the SGWB energy density spectrum from $\mathrm{PBH}$ binaries, compares it to that from astrophysical BBHs and discusses the detectability for future GW detectors. The PBH background was shown to have the same power law spectrum $f^{2 / 3}$ as that from astrophysical BBHs in the Advanced LIGO sensitivity band. Moreover, it has been suggested that the SGWB can be used to investigate the PBH abundance. Here, we consider the constraints on the PBH abundance using the SGWB in a different PBH binary formation framework by Sasaki et al. [10] which produces binaries in the early Universe, as opposed to that used by Ref. [43] which forms binaries in the late Universe. Since the PBHs in the early Universe are distributed more densely, the Sasaki et al. [10] framework has a larger merger rate, leading to a stronger SGWB amplitude compared with Ref. [43].

Constraining the primordial black hole abundance with the stochastic gravitational-wave background.- Since the first Advanced LIGO observation run did not find evidence for a SGWB signal [42], we can use the nondetection to constrain the maximum SGWB energy density spectrum $\Omega_{\mathrm{GW}}^{\max }(\nu)$, and to further constrain the maximum $\mathrm{PBH}$ abundance $f_{\max }$ by equating

$$
\Omega_{\mathrm{GW}}^{\max }(\nu)=\Omega_{\mathrm{GW}}\left(\nu ; M_{\mathrm{PBH}}, f_{\max }\right),
$$

thereby giving a upper limit $f_{\max }$ on the $\mathrm{PBH}$ abundance for different $M_{\mathrm{PBH}}$. Taking advantage of the unique ob- servational window from GW, the SGWB yields a new independent constraint on the properties of $\mathrm{PBHs}$ which we can compare to other methods, such as the lensing of stars and quasars, dynamics of dwarf galaxies, large scale structure and accretion effects on the CMB [21].

Figure 1 shows the current upper limit in the $f-M_{\mathrm{PBH}}$ plane from Advanced LIGO's first observation run (O1, 2015-16, black solid), and the expected constraints from the second observation run (O2, 2016-17, black dashed) and the fifth observation run (O5, 2020-22, dot dashed). For comparison, constraints on $f$ from the EROS-OGLE

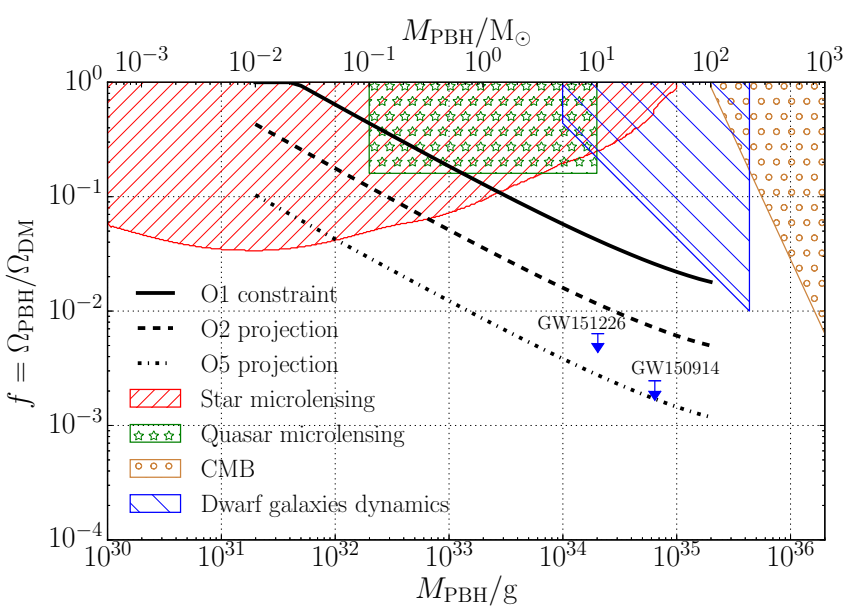

FIG. 1. The constraints on the PBH fraction in dark matter $f_{\text {max }}$ versus the $\mathrm{PBH}$ mass $M_{\mathrm{PBH}}$ from the nondetection of the SGWB from Advanced LIGO's O1 and the expected constraints based on the $\mathrm{O} 2$ and $\mathrm{O} 5$ projected sensitivities. These constraints are compared to those from star microlensing [15], quasar microlensing [16], dynamics of dwarf galaxies [63] and accretion effects on CMB [19]. The local merger rate for GW150914- and GW151226-like BBHs can also constrain the $\mathrm{PBH}$ abundance with corresponding mass.

microlensing of stars (This result is obtained by combining EROS and OGLE detections and achieved tighter constraints by assuming that a few positive detections from OGLE are explained by self-lensing.) [15] , microlensing of quasars [16], dynamics of dwarf galaxies [63], and accretion effect on CMB [19] are also plotted.

In addition, the inferred local merger rates associated with the GW150914- and GW151226 like BBHs can also constrain the abundance of PBHs. Since we adopt a delta PBH mass distribution following Sasaki et al. [10] given the large theoretical uncertainty, for consistency, we also consider the LIGO's local merger rate estimated by assuming all the black holes have the same mass as detected rather than an extended distribution. By imposing the condition that $R_{\mathrm{PBH}}\left(z=0 ; M_{\mathrm{PBH}}, f_{\max }\right)$ cannot exceed the maximum of the estimated local merger rate, an upper limit on the $\mathrm{PBH}$ abundance $f_{\max }$ can be given with corresponding $M_{\mathrm{PBH}}$, as shown in Fig. 1.

We see that up to now microlensing gives the tightest 
constraints in the mass range $10^{-3} M_{\odot} \lesssim M_{\mathrm{PBH}} \lesssim 1 M_{\odot}$. The new upper limit given by SGWB from Advanced LIGO's O1 gives the best constraint on the PBH abundance in the mass range $1 M_{\odot} \lesssim M_{\mathrm{PBH}} \lesssim 100 M_{\odot}(\mathrm{PBH}$ binaries with masses higher than $\sim O\left(10^{2}\right) M_{\odot}$ would have lower cut-off frequency, thus would evade the frequency band of Advanced LIGO), pushing the previous microlensing and dwarf galaxy dynamics constraint tighter by 1 order of magnitude. Future observing runs of Advanced LIGO are expected to improve the constraint $f_{\max }$ further to $O\left(10^{-3}\right)$.

Conversely, we can compare the SGWB spectra from the current constraints to the expected Advanced LIGO sensitivities. Fig. 2 shows the SGWB spectra from binary PBH mergers for different chirp masses using the current most stringent constraints of the PBH abundance. Here, the chirp mass is defined by $M_{c}=\left(m_{1} m_{2}\right)^{3 / 5} /\left(m_{1}+\right.$ $\left.m_{2}\right)^{1 / 5}$, where $m_{1}$ and $m_{2}$ are the component mass of BBHs. Thus, $M_{c}=M_{\mathrm{PBH}} / 2^{1 / 5}$ for PBHs of a fixed mass. In Fig. 2, the black curves denote the $1 \sigma$ sensitivity of the LIGO-Virgo network expected for two first observing runs O1 (black solid) and O2 (black dashed), and at the design sensitivity in O5 (black dot dashed) [41, 64]. The sensitivity curve is calculated in the context of the cross correlation statistic method [60] with one year of integration, and the coincident duty cycle is $30 \%$ for $\mathrm{O} 1$ and $50 \%$ for $\mathrm{O} 2$ and $\mathrm{O} 5$. If a model-predicting spectrum intersects a black curve, then it has an expected signalto-noise ratio greater or equal than 1 .

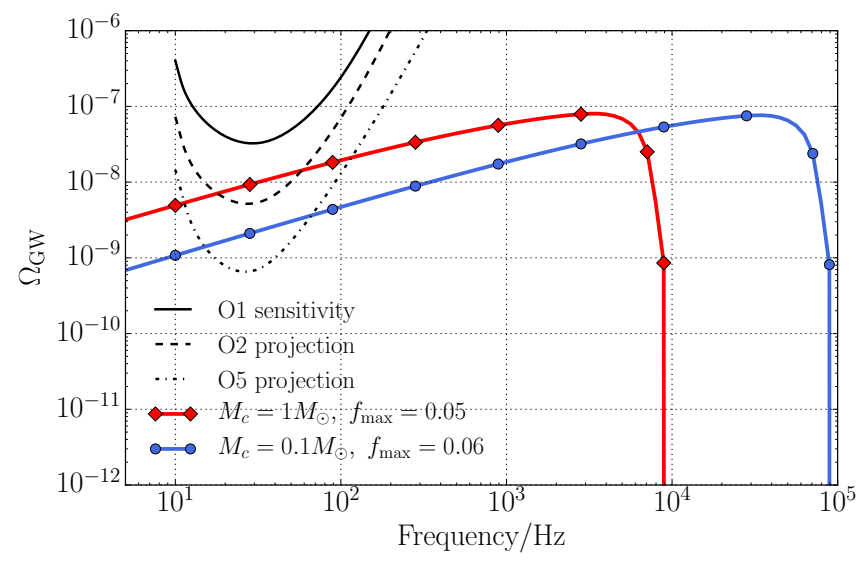

FIG. 2. The SGWB spectra from subsolar mass binary $\mathrm{PBH}$ mergers at the current best constraints from stellar microlensing. Nondetection in Advanced LIGO's O2 can further constrain the existence of subsolar mass PBHs.

From Fig. 2 we can see that the SGWB generated by subsolar mass PBHs has the opportunity to be detected by upcoming Advanced LIGO observing runs. Therefore, the SGWB provides a possible way to explore the existence of subsolar mass PBHs, which would be a smoking gun for the existence of $\mathrm{PBHs}$ since subsolar mass BHs are not expected to be of a stellar origin. However, a decisive evidence for PBHs would need the detection of SGWB at high frequency, which is beyond the scope of Advanced LIGO. Nevertheless, SGWB provides a complementary channel to investigate the properties of subsolar mass BHs, even if their GW signal is too weak to be individually resolved.

Discussion.--In this work, we place a novel constraint on the PBH abundance in dark matter in the mass range $0.01 M_{\odot}-100 M_{\odot}$ using the current nondetection of SGWB from Advanced LIGO's first observation run. As a new observational window, the constraint from SGWB is better than other methods such as microlensing and dwarf galaxy dynamics by 1 order of magnitude in the mass range $1 M_{\odot} \lesssim M_{\mathrm{PBH}} \lesssim 100 M_{\odot}$. Finally, we also find that the current most stringent constraints on the abundance of subsolar mass PBHs can give a measurable SGWB by future Advanced LIGO observing runs.

The coalescence of a pair of $\mathrm{PBHs}$ produces a $\mathrm{BH}$ of higher mass, and this evolution of the mass distribution has an effect on the SGWB spectrum. Nevertheless, at the matter-radiation equality epoch $z_{\text {eq }}$, only a pair of PBHs that satisfies $x<f^{1 / 3} \bar{x}$ can form a binary [10], where $x$ is the physical separation between two neighboring PBHs. This means that the fraction of PBHs that can form binaries in the total $\mathrm{PBH}$ population is $x_{\max }^{3} / \bar{x}^{3} \simeq f$. Thus, the fraction of subsequent moremassive PBH binaries in the original population of $\mathrm{PBH}$ binaries is also given by $f$. From Fig. 1, the typical value of $f_{\max }$ is of the order of $O(0.01)$. However, the mass doubling effect will only contribute an extra factor of $2^{5 / 3} \sim 3$ to the GW energy density spectrum $\left(d E / d \nu \propto M_{c}^{5 / 3}\right)$. Therefore, we expect that the evolution of the mass distribution has a negligible effect on the SGWB in this work's scenario. However, we also notice that PBHs may be clustered in the late Universe, boosting the formation rate of more-massive $\mathrm{PBH}$ binaries. The effect of such clustering is beyond the scope of this Letter and is left for a future work.

Another consideration is that $\mathrm{PBH}$ binaries may be formed with highly eccentric orbits, and these binaries could preserve the eccentricity until merger if they coalesce on timescales within years or less [44, 65]. In this work, the contribution of PBH mergers to the SGWB spectrum in the Advanced LIGO sensitive frequency band comes from the redshift $z<\nu_{\text {cut }} / \nu-1$. Compared with the binary formation epoch, which is earlier than the matter-radiation epoch $z_{\text {eq }}$, the $\mathrm{PBH}$ binaries are expected to have enough time to circularize the orbits. Therefore, we assume that the effects of eccentricity are negligible when calculating the SGWB in this work. However, when considering a lower frequency band, one should include the influence of the gravitational-wave emission from eccentric binaries (see, e.g., Ref. [66-72]) on the SGWB.

Finally, our results depend on the merger rate of Sasaki et al. [10], which in turn assumes that binaries formed 
at early times and not merged yet survive until $z=0$. The PBH binary formation and evolution are still under active investigation (see, e.g. Ref. [73-75]). Future GW measurements will further shed light on the $\mathrm{PBH}$ scenario.

Recently, three more GW events from BBH merger, GW170104, GW170608 and GW170814, were announced during the second Advanced LIGO-Virgo observation run [76-78]. In the absence of a publicly available event rate statement from each event alone and SGWB results for the second observation run, we leave this analysis for future work.

S.W. is partially supported by the funding from the Research Grants Council of Hong Kong (Project No. 14301214 and ECS No. 2191110). Y.-F.W and T.G.F.L were partially supported by a grant from the Research Grants Council of Hong Kong (Project No. CUHK 14310816) and by the Direct Grant for Research from the Research Committee of the Chinese University of Hong Kong. Q.-G.H. is supported by grants from NSFC (Grants No. 11335012, No. 11575271, and No. 11690021), and the Top-Notch Young Talents Program of China, and is partly supported by the Key Research Program of Frontier Sciences, CAS. Q.-G.H. also acknowledges the hospitality of HKUST during the last stage of this project.

[1] B. P. Abbott et al. (Virgo, LIGO Scientific), "Observation of Gravitational Waves from a Binary Black Hole Merger," Phys. Rev. Lett. 116, 061102 (2016), arXiv:1602.03837 [gr-qc].

[2] B. P. Abbott et al. (Virgo, LIGO Scientific), "GW151226: Observation of Gravitational Waves from a 22-SolarMass Binary Black Hole Coalescence," Phys. Rev. Lett. 116, 241103 (2016), arXiv:1606.04855 [gr-qc].

[3] B. P. Abbott et al., "Binary Black Hole Mergers in the first Advanced LIGO Observing Run," Phys. Rev. X6, 041015 (2016), arXiv:1606.04856 [gr-qc].

[4] B. P. Abbott et al. (Virgo, LIGO Scientific), "Astrophysical Implications of the Binary Black-Hole Merger GW150914," Astrophys. J. 818, L22 (2016), arXiv:1602.03846 [astro-ph.HE].

[5] Krzysztof Belczynski, Daniel E. Holz, Tomasz Bulik, and Richard O'Shaughnessy, "The first gravitational-wave source from the isolated evolution of two 40-100 Msun stars," Nature 534, 512 (2016), arXiv:1602.04531 [astroph.HE].

[6] K. Belczynski, M. Dominik, T. Bulik, R. O'Shaughnessy, C. L. Fryer, and D. E. Holz, "The effect of metallicity on the detection prospects for gravitational waves," Astrophys. J. 715, L138 (2010), arXiv:1004.0386 [astroph.HE].

[7] M. Coleman Miller, "Implications of the gravitational wave event GW150914," Gen. Rel. Grav. 48, 95 (2016), arXiv:1606.06526 [astro-ph.HE].

[8] Simeon Bird, Ilias Cholis, Julian B. Muñoz, Yacine Ali-
Haïmoud, Marc Kamionkowski, Ely D. Kovetz, Alvise Raccanelli, and Adam G. Riess, "Did ligo detect dark matter?" Phys. Rev. Lett. 116, 201301 (2016).

[9] Sebastien Clesse and Juan García-Bellido, "The clustering of massive Primordial Black Holes as Dark Matter: measuring their mass distribution with Advanced LIGO," Phys. Dark Univ. 10, 002 (2016), arXiv:1603.05234 [astro-ph.CO].

[10] Misao Sasaki, Teruaki Suyama, Takahiro Tanaka, and Shuichiro Yokoyama, "Primordial Black Hole Scenario for the Gravitational-Wave Event GW150914," Phys. Rev. Lett. 117, 061101 (2016), arXiv:1603.08338 [astroph.CO].

[11] Lu Chen, Qing-Guo Huang, and Ke Wang, "Constraint on the abundance of primordial black holes in dark matter from Planck data," (2016), arXiv:1608.02174 [astroph.CO].

[12] A. Kashlinsky, "LIGO gravitational wave detection, primordial black holes and the near-IR cosmic infrared background anisotropies," Astrophys. J. 823, L25 (2016), arXiv:1605.04023 [astro-ph.CO].

[13] Nicola Bartolo et al., "Science with the space-based interferometer LISA. IV: Probing inflation with gravitational waves," (2016), arXiv:1610.06481 [astro-ph.CO].

[14] Ilias Cholis, "On the Gravitational Wave Background from Black Hole Binaries after the First LIGO Detections," (2016), arXiv:1609.03565 [astro-ph.HE].

[15] S. Calchi Novati, S. Mirzoyan, Ph. Jetzer, and G. Scarpetta, "Microlensing towards the SMC: a new analysis of OGLE and EROS results," Mon. Not. Roy. Astron. Soc. 435, 1582 (2013), arXiv:1308.4281 [astro-ph.GA].

[16] E. Mediavilla, J. A. Munoz, E. Falco, V. Motta, E. Guerras, H. Canovas, C. Jean, A. Oscoz, and A. M. Mosquera, "Microlensing-Based Estimate of the Mass Fraction in Compact Objects in Lens," Astrophys. J. 706, 1451-1462 (2009), arXiv:0910.3645 [astro-ph.CO].

[17] Anne M. Green, "Microlensing and dynamical constraints on primordial black hole dark matter with an extended mass function," Phys. Rev. D94, 063530 (2016), arXiv:1609.01143 [astro-ph.CO].

[18] G. Chapline and P. H. Frampton, "A new direction for dark matter research: intermediate mass compact halo objects," JCAP 1611, 042 (2016), arXiv:1608.04297 [grqc].

[19] Yacine Ali-Haïmoud and Marc Kamionkowski, "Cosmic microwave background limits on accreting primordial black holes," Phys. Rev. D 95, 043534 (2017).

[20] Katelin Schutz and Adrian Liu, "Pulsar timing can constrain primordial black holes in the LIGO mass window," (2016), arXiv:1610.04234 [astro-ph.CO].

[21] Bernard Carr, Florian Kuhnel, and Marit Sandstad, "Primordial Black Holes as Dark Matter," Phys. Rev. D94, 083504 (2016), arXiv:1607.06077 [astro-ph.CO].

[22] Andi Tan et al. (PandaX-II), "Dark Matter Results from First 98.7 Days of Data from the PandaX-II Experiment," Phys. Rev. Lett. 117, 121303 (2016), arXiv:1607.07400 [hep-ex].

[23] D. S. Akerib et al. (LUX), "Improved Limits on Scattering of Weakly Interacting Massive Particles from Reanalysis of 2013 LUX Data," Phys. Rev. Lett. 116, 161301 (2016), arXiv:1512.03506 [astro-ph.CO].

[24] ATLAS Collaboration and CMS Collaboration (ATLAS, CMS), "Combination of results from the ATLAS and CMS experiments on anomalous triple gauge couplings 
in $\mathrm{ZZ}$ production from pp collisions at a centre-of-mass energy of $7 \mathrm{TeV}$ at the LHC," (2016).

[25] L. Accardo et al. (AMS), "High Statistics Measurement of the Positron Fraction in Primary Cosmic Rays of 0.5-500 GeV with the Alpha Magnetic Spectrometer on the International Space Station," Phys. Rev. Lett. 113, 121101 (2014).

[26] M. Ackermann et al. (Fermi-LAT), "Measurement of separate cosmic-ray electron and positron spectra with the Fermi Large Area Telescope," Phys. Rev. Lett. 108, 011103 (2012), arXiv:1109.0521 [astro-ph.HE].

[27] Jonathan L. Feng, "Dark Matter Candidates from Particle Physics and Methods of Detection," Ann. Rev. Astron. Astrophys. 48, 495-545 (2010), arXiv:1003.0904 [astro-ph.CO].

[28] Stephen Hawking, "Gravitationally collapsed objects of very low mass," Mon. Not. Roy. Astron. Soc. 152, 75 (1971).

[29] Bernard J. Carr and S. W. Hawking, "Black holes in the early Universe," Mon. Not. Roy. Astron. Soc. 168, 399415 (1974).

[30] Juan Garcia-Bellido, Andrei D. Linde, and David Wands, "Density perturbations and black hole formation in hybrid inflation," Phys. Rev. D54, 6040-6058 (1996), arXiv:astro-ph/9605094 [astro-ph].

[31] Sébastien Clesse and Juan García-Bellido, "Massive primordial black holes from hybrid inflation as dark matter and the seeds of galaxies," Phys. Rev. D 92, 023524 (2015).

[32] A. D. Dolgov and S. I. Blinnikov, "Stars and Black Holes from the very Early Universe," Phys. Rev. D89, 021301 (2014), arXiv:1309.3395 [astro-ph.CO].

[33] Takashi Nakamura, Misao Sasaki, Takahiro Tanaka, and Kip S. Thorne, "Gravitational waves from coalescing black hole MACHO binaries," Astrophys. J. 487, L139L142 (1997), arXiv:astro-ph/9708060 [astro-ph].

[34] Tania Regimbau, "The astrophysical gravitational wave stochastic background," Res. Astron. Astrophys. 11, 369-390 (2011), arXiv:1101.2762 [astro-ph.CO].

[35] Xing-Jiang Zhu, E. Howell, T. Regimbau, D. Blair, and Zong-Hong Zhu, "Stochastic Gravitational Wave Background from Coalescing Binary Black Holes," Astrophys. J. 739, 86 (2011), arXiv:1104.3565 [gr-qc].

[36] C. Wu, V. Mandic, and T. Regimbau, "Accessibility of the Gravitational-Wave Background due to Binary Coalescences to Second and Third Generation Gravitational-Wave Detectors," Phys. Rev. D85, 104024 (2012), arXiv:1112.1898 [gr-qc].

[37] Xing-Jiang Zhu, Eric J. Howell, David G. Blair, and Zong-Hong Zhu, "On the gravitational wave background from compact binary coalescence in the band of groundbased interferometers," MNRAS, 431: 882-899 (2013), arXiv:1209.0595 [gr-qc].

[38] Cheng-Jian Wu, Vuk Mandic, and Tania Regimbau, "Accessibility of the stochastic gravitational wave background from magnetars to the interferometric gravitational wave detectors," Phys. Rev. D87, 042002 (2013).

[39] S. Marassi, R. Schneider, G. Corvino, V. Ferrari, and S. Portegies Zwart, "Imprint of the merger and ring-down on the gravitational wave background from black hole binaries coalescence," Phys. Rev. D 84, 124037 (2011).

[40] Pablo A. Rosado, "Gravitational wave background from binary systems," Phys. Rev. D84, 084004 (2011), arXiv:1106.5795 [gr-qc].
[41] B. P. Abbott et al. (Virgo, LIGO Scientific), "GW150914: Implications for the stochastic gravitational wave background from binary black holes," Phys. Rev. Lett. 116, 131102 (2016), arXiv:1602.03847 [gr-qc].

[42] Benjamin P. Abbott et al. (Virgo, LIGO Scientific), "Upper Limits on the Stochastic Gravitational-Wave Background from Advanced LIGO's First Observing Run," Phys. Rev. Lett. 118, 121101 (2017), arXiv:1612.02029 [gr-qc].

[43] Vuk Mandic, Simeon Bird, and Ilias Cholis, "Stochastic Gravitational-Wave Background due to Primordial Binary Black Hole Mergers," Phys. Rev. Lett. 117, 201102 (2016), arXiv:1608.06699 [astro-ph.CO].

[44] Sebastien Clesse and Juan García-Bellido, "Detecting the gravitational wave background from primordial black hole dark matter," (2016), arXiv:1610.08479 [astroph.CO].

[45] Kunihito Ioka, Takeshi Chiba, Takahiro Tanaka, and Takashi Nakamura, "Black hole binary formation in the expanding universe: Three body problem approximation," Phys. Rev. D58, 063003 (1998), arXiv:astroph/9807018 [astro-ph].

[46] P. A. R. Ade et al. (Planck), "Planck 2015 results. XIII. Cosmological parameters," Astron. Astrophys. 594, A13 (2016), arXiv:1502.01589 [astro-ph.CO].

[47] Masahiro Kawasaki, Naoya Kitajima, and Tsutomu T. Yanagida, "Primordial black hole formation from an axionlike curvaton model," Phys. Rev. D87, 063519 (2013), arXiv:1207.2550 [hep-ph].

[48] K. Jedamzik and Jens C. Niemeyer, "Primordial black hole formation during first order phase transitions," Phys. Rev. D59, 124014 (1999), arXiv:astro-ph/9901293 [astro-ph].

[49] Hideo Kodama, Misao Sasaki, and Katsuhiko Sato, "Abundance of Primordial Holes Produced by Cosmological First Order Phase Transition," Prog. Theor. Phys. 68, 1979 (1982).

[50] M. Yu. Khlopov, R. V. Konoplich, S. G. Rubin, and A. S. Sakharov, "First-order phase transitions as a source of black holes in the early universe," Grav. Cosmol. 6, 153156 (2000).

[51] Bernard J. Carr, "The Primordial black hole mass spectrum," Astrophys. J. 201, 1-19 (1975).

[52] S. W. Hawking, "Black Holes From Cosmic Strings," Phys. Lett. B231, 237-239 (1989).

[53] Martti Raidal, Ville Vaskonen, and Hardi Veermäe, "Gravitational Waves from Primordial Black Hole Mergers," JCAP 1709, 037 (2017), arXiv:1707.01480 [astroph.CO].

[54] E. Vangioni, K. A. Olive, T. Prestegard, J. Silk, P. Petitjean, and V. Mandic, "The Impact of Star Formation and Gamma-Ray Burst Rates at High Redshift on Cosmic Chemical Evolution and Reionization," Mon. Not. Roy. Astron. Soc. 447, 2575 (2015), arXiv:1409.2462 [astroph.GA].

[55] Takashi Nakamura et al., "Pre-DECIGO can get the smoking gun to decide the astrophysical or cosmological origin of GW150914-like binary black holes," 2016 , 093E01 (2016), arXiv:1607.00897 [astro-ph.HE].

[56] Gerhard Heinzel and Karsten Danzmann, "LISA in 2012 and Beyond: 20 Years After the First Proposal," Proceedings, Relativity and Gravitation: Perspectives 100 years after Einstein's stay in Prague: Prague, Czech Republic, June 25-29, 2012, Fundam. Theor. Phys. 177, 477-481 
(2014).

[57] S. Kawamura et al., "The Japanese space gravitational wave antenna DECIGO," Gravitational waves. Proceedings, 6th Edoardo Amaldi Conference, Amaldi6, Bankoku Shinryoukan, June 20-24, 2005, Class. Quant. Grav. 23, S125-S132 (2006).

[58] G. M. Harry, P. Fritschel, D. A. Shaddock, W. Folkner, and E. S. Phinney, "Laser interferometry for the big bang observer," Class. Quant. Grav. 23, 4887-4894 (2006), [Erratum: Class. Quant. Grav.23,7361(2006)].

[59] Alvise Raccanelli, Ely D. Kovetz, Simeon Bird, Ilias Cholis, and Julian B. Munoz, "Determining the progenitors of merging black-hole binaries," Phys. Rev. D94, 023516 (2016), arXiv:1605.01405 [astro-ph.CO].

[60] Bruce Allen and Joseph D. Romano, "Detecting a stochastic background of gravitational radiation: Signal processing strategies and sensitivities," Phys. Rev. D59, 102001 (1999), arXiv:gr-qc/9710117 [gr-qc].

[61] P. Ajith, S. Babak, Y. Chen, M. Hewitson, B. Krishnan, A. M. Sintes, J. T. Whelan, B. Brügmann, P. Diener, N. Dorband, J. Gonzalez, M. Hannam, S. Husa, D. Pollney, L. Rezzolla, L. Santamaría, U. Sperhake, and J. Thornburg, "Template bank for gravitational waveforms from coalescing binary black holes: Nonspinning binaries," Phys. Rev. D 77, 104017 (2008).

[62] P. Ajith, M. Hannam, S. Husa, Y. Chen, B. Brügmann, N. Dorband, D. Müller, F. Ohme, D. Pollney, C. Reisswig, L. Santamaría, and J. Seiler, "Inspiral-mergerringdown waveforms for black-hole binaries with nonprecessing spins," Phys. Rev. Lett. 106, 241101 (2011).

[63] Savvas M. Koushiappas and Abraham Loeb, "Dynamics of Dwarf Galaxies Disfavor Stellar-Mass Black Holes as Dark Matter," Phys. Rev. Lett. 119, 041102 (2017), arXiv:1704.01668 [astro-ph.GA].

[64] Eric Thrane and Joseph D. Romano, "Sensitivity curves for searches for gravitational-wave backgrounds," Phys. Rev. D88, 124032 (2013), arXiv:1310.5300 [astro-ph.IM].

[65] Ilias Cholis, Ely D. Kovetz, Yacine Ali-Haïmoud, Simeon Bird, Marc Kamionkowski, Julian B. Muñoz, and Alvise Raccanelli, "Orbital eccentricities in primordial black hole binaries," Phys. Rev. D 94, 084013 (2016).

[66] Nicolas Yunes, K. G. Arun, Emanuele Berti, and Clifford M. Will, "Post-Circular Expansion of Eccentric Binary Inspirals: Fourier-Domain Waveforms in the Stationary Phase Approximation," Phys. Rev. D80, 084001 (2009), [Erratum: Phys. Rev.D89,no.10,109901(2014)], arXiv:0906.0313 [gr-qc].

[67] E. A. Huerta, Prayush Kumar, Sean T. McWilliams, Richard O'Shaughnessy, and Nicolás Yunes, "Accurate and efficient waveforms for compact binaries on eccentric orbits," Phys. Rev. D 90, 084016 (2014).

[68] Balázs Mikóczi, Péter Forgács, and Mátyás Vasúth, "First order post-newtonian gravitational waveforms of binaries on eccentric orbits with hansen coefficients," Phys. Rev. D 92, 044038 (2015).

[69] Sashwat Tanay, Maria Haney, and Achamveedu Gopakumar, "Frequency and time domain inspiral templates for comparable mass compact binaries in eccentric orbits," Phys. Rev. D93, 064031 (2016), arXiv:1602.03081 [grqc].

[70] Chandra Kant Mishra, K. G. Arun, and Bala R. Iyer, "Third post-Newtonian gravitational waveforms for compact binary systems in general orbits: Instantaneous terms," Phys. Rev. D91, 084040 (2015), arXiv:1501.07096 [gr-qc].

[71] E. A. Huerta, Prayush Kumar, Bhanu Agarwal, Daniel George, Hsi-Yu Schive, Harald P. Pfeiffer, Roland Haas, Wei Ren, Tony Chu, Michael Boyle, Daniel A. Hemberger, Lawrence E. Kidder, Mark A. Scheel, and Bela Szilagyi, "Complete waveform model for compact binaries on eccentric orbits," Phys. Rev. D 95, 024038 (2017).

[72] Siyuan Chen, Alberto Sesana, and Walter Del Pozzo, "Efficient computation of the gravitational wave spectrum emitted by eccentric massive black hole binaries in stellar environments," (2016), arXiv:1612.00455 [astroph.CO].

[73] Kimitake Hayasaki, Keitaro Takahashi, Yuuiti Sendouda, and Shigehiro Nagataki, "Rapid merger of binary primordial black holes: An implication for GW150914," Publ. Astron. Soc. Jap. 68, 66 (2016), arXiv:0909.1738 [astroph.CO].

[74] Yu. N. Eroshenko, "Formation of PBHs binaries and gravitational waves from their merge," (2016), arXiv:1604.04932 [astro-ph.CO].

[75] Yacine Ali-Haïmoud, Ely D. Kovetz, and Marc Kamionkowski, "The merger rate of primordial-blackhole binaries," (2017), arXiv:1709.06576 [astro-ph.CO].

[76] Benjamin P. Abbott et al. (VIRGO, LIGO Scientific), "GW170104: Observation of a 50-Solar-Mass Binary Black Hole Coalescence at Redshift 0.2," Phys. Rev. Lett. 118, 221101 (2017), arXiv:1706.01812 [gr-qc].

[77] B. P. Abbott et al. (Virgo, LIGO Scientific), "GW170608: Observation of a 19-solar-mass Binary Black Hole Coalescence," (2017), arXiv:1711.05578 [astro-ph.HE].

[78] B. P. Abbott et al. (Virgo, LIGO Scientific), "GW170814: A Three-Detector Observation of Gravitational Waves from a Binary Black Hole Coalescence," Phys. Rev. Lett. 119, 141101 (2017), arXiv:1709.09660 [gr-qc]. 